\title{
Enhanced magnetic performance of bulk nanocrystalline MnAl-C prepared by high pressure compaction of gas atomized powders
}

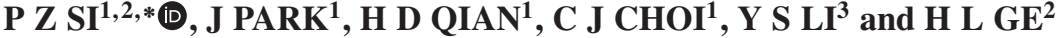 \\ ${ }^{1}$ Powder and Ceramic Division, Korea Institute of Materials Science, Changwon 51508, Republic of Korea \\ ${ }^{2}$ College of Materials Science and Engineering, China Jiliang University, Hangzhou 310018, People's Republic of China \\ ${ }^{3}$ Engineering Ceramics Research Group, Korea Institute of Materials Science, Changwon 51508, Republic of Korea \\ *Author for correspondence (pzsi@mail.com)
}

MS received 5 May 2018; accepted 12 September 2018; published online 27 March 2019

\begin{abstract}
High density MnAl-C magnets with enhanced coercivity and remanent magnetization were prepared by highpressure compaction of the $\tau$-phase obtained by annealing the as-prepared gas-atomized powders, which are spherical in shape with size in the range of 1-7 $\mu \mathrm{m}$. The as-prepared gas-atomized powders were composed of $\varepsilon$ - as the major phase and $\gamma_{2}$ - as the minor phase. The massive phase transformation of $\varepsilon \rightarrow \tau$ in the gas-atomized powders occurs at $720 \mathrm{~K}$ and accomplishes at $806 \mathrm{~K}$, both of which are lower than those of the water-quenched $\varepsilon$-MnAl-C alloys with the same composition. An optimized temperature of $760 \mathrm{~K}$, at which the decomposition of metastable $\tau$-phase was minimized, was selected to prepare the ferromagnetic $\tau$ - from the $\varepsilon$-phase. The spherical $\tau$-phase powders were pressed at room temperature into two dimensional plates that stack along the direction of compaction, forming high density (98.6\%) bulk magnets that exhibit larger coercivity and higher remanent magnetization than that of the $\tau$-phase powders. The grain size of the compacted samples was observed to be in the range of $10-100 \mathrm{~nm}$. The coercivity $(0.34 \mathrm{~T})$ of the dense samples is twice as large as that of the $\tau$-phase powders, owing to the refined grain size and enlarged dislocation density resulting from high-pressure compaction.
\end{abstract}

Keywords. Gas atomization; high pressure synthesis; MnAl; nanocrystalline; magnetic properties.

\section{Introduction}

The existence of a ferromagnetic phase in the $\mathrm{Mn}-\mathrm{Al}$ system has been pointed out for more than 110 years [1]. The preparation of tetragonal ferromagnetic phase containing about 54 at.\% manganese had attracted intensive research interests in the 1960-1970s [2,3]. However, the discovery of $\mathrm{Nd}-\mathrm{Fe}-\mathrm{B}$ soon interrupted the research on MnAl-based magnets, which became attractive again recently due to the low cost of MnAl alloys and its medium magnetic performance filling the gap between ferrites and $\mathrm{Nd}-\mathrm{Fe}-\mathrm{B}$ [4-7]. Unfortunately, it has been not easy to produce large coercivity $\left(H_{\mathrm{c}}\right)$ in the MnAl-based magnets. Moreover, the ferromagnetic $\mathrm{Mn}-\mathrm{Al}$ binary alloys are meta-stable and may decompose during high temperature processing. Therefore, carbon is usually employed to improve the stability of the meta-stable $\mathrm{Mn}-\mathrm{Al}$ magnetic materials. The coercivity of the $\mathrm{C}$-doped $\mathrm{MnAl}$ is usually slightly lower than that of the C-free $\mathrm{MnAl}$. In addition, the gas-atomized powders usually show an enhanced coercivity in comparison with the as-cast $\mathrm{MnAl}$ alloys [8,9]. After milling and annealing, the gas-atomized $\mathrm{Mn}_{54} \mathrm{Al}_{46}$ powders showed a coercivity up to $0.49 \mathrm{~T}$ [10]. However, the ball-milled powders with large coercivities usually exhibit significantly reduced saturation magnetization $\left(M_{\mathrm{s}}\right)[9,10]$. In this work, high pressure compaction was employed to reduce the grain size of $\mathrm{MnAl}-\mathrm{C}$. An enhanced $H_{\mathrm{c}}$ and an enhanced remanent magnetization $\left(M_{\mathrm{r}}\right)$ were achieved in bulk MnAl-C without significant reduction of the $M_{\mathrm{s}}$.

Several methods such as hot extrusion, hot deformation, hot compaction, spark plasma sintering, equal channel angular extrusion processing and microwave sintering have been employed to fabricate bulk MnAl-based magnets [6,11-14]. The traditional methods to make bulk MnAl-based magnetic materials usually involve high temperature processes which may result in partial decomposition of the $\tau$-phase and thus a reduced magnetization. Tyrman et al [15] prepared $\mathrm{Mn}-\mathrm{Al}-\mathrm{C}$ by high pressure (up to $400 \mathrm{MPa}$ ) spark plasma sintering at $\sim 550^{\circ} \mathrm{C}$, at which the precipitation of a few percent of $\beta$-phase was observed. The best properties of bulk $\mathrm{Mn}-\mathrm{Al}-\mathrm{C}$ so far is reported in the hot-extruded samples with an $H_{\mathrm{c}}$ of $\sim 0.3 \mathrm{~T}$ and a $(B H)_{\max }$ of $\sim 7$ MGOe [11]. A cold-rolling and swaging process was recently employed to enhance the coercivity of the MnAl-C magnetic materials $[16,17]$. In this work, high density MnAl-C magnets with enhanced $H_{\mathrm{c}}$ and enhanced $M_{\mathrm{r}}$ were prepared by using a high-pressure compaction process (up to $3.1 \mathrm{GPa}$ ) at room temperature, which avoids the high temperature process that may result in the decomposition of the ferromagnetic $\tau$-phase. 


\section{Experimental}

The MnAl-C alloyed ingots with an atomic ratio of $\mathrm{Mn}: \mathrm{Al}: \mathrm{C}=54: 46: 2.44$ were prepared by using the traditional induction melting method with precursors of manganese pieces (99.95\% purity), aluminium pellets ( $99.999 \%$ purity) and high purity carbon powders. In analogy to the preparation of $\mathrm{Mn}-\mathrm{Al}$ powders [18], the gas-atomized $\mathrm{MnAl}-\mathrm{C}$ powders were obtained by ejecting the melted alloys through a nozzle with high pressure nitrogen gas in a vacuumed chamber. Then, the gas-atomized powders in the chamber were collected for subsequent experiments. The phase transformation temperatures of the gas-atomized powders were determined by measuring the magnetization $v s$. temperature $(M-T)$ curves under an applied field of $2 \mathrm{~T}$ with increasing temperature $(20 \mathrm{~K}$ $\left.\mathrm{min}^{-1}\right)$ from 300 to $900 \mathrm{~K}$. The Curie temperature $\left(T_{\mathrm{c}}\right)$ of the samples was measured under $10 \mathrm{mT}$ with decreasing temperature $\left(20 \mathrm{~K} \mathrm{~min}^{-1}\right)$ from $900 \mathrm{~K}$. The magnetic hysteresis loops of the samples were recorded at $290 \mathrm{~K}$ in fields up to $8 \mathrm{~T}$. The $\tau$-phase $\mathrm{MnAl}-\mathrm{C}$ powders were produced by annealing the asprepared gas-atomized powders at $760 \mathrm{~K}$ for $20 \mathrm{~min}$ and then sealed in a stainless-steel tube. Then, the bulk samples were prepared by compacting the $\tau$-phase $\mathrm{MnAl}-\mathrm{C}$ powders in the tube under a pressure of $\sim 1.6-3.1 \mathrm{GPa}$. The density of the bulk samples was measured by using a hydrostatic balance, while the structural evolution of the samples was determined by using X-ray powder diffraction (XRD) with $\mathrm{CuK} \alpha$ radiation. The morphology of the gas-atomized powders and the microstructure of the high pressure compacted bulk samples were analysed by using scanning electron microscopy (SEM) and transmission electron microscopy (TEM), respectively. The thin foil of the bulk samples for TEM observations were prepared by using a focussed ion beam work station. The magnetic properties of the samples were measured by using a Quantum Design physical properties measurement system.

\section{Results and discussion}

\subsection{Morphology of gas-atomized powders}

Figure 1 shows the morphology of the gas-atomized MnAl$\mathrm{C}$ powders. An ideal spherical shape of the powders was observed and this was ascribed to the surface tension effect of the tiny liquid MnAl-C droplets when sprayed with high pressure $\mathrm{N}_{2}$ and solidified at a high cooling rate. The typical size of the gas-atomized powders lies in the range of 1-7 $\mu \mathrm{m}$ and the size distribution of the powders is relatively narrow. Note that the morphology of the gas-atomized powders shows no changes after heat treatment due to the low treating temperatures.

\subsection{Structural evolution detected by XRD}

XRD patterns of the gas-atomized MnAl-C powders and their products after annealing at $760 \mathrm{~K}$ for $20 \mathrm{~min}$ are shown in figure 1 . The reflections of the samples have been normalized

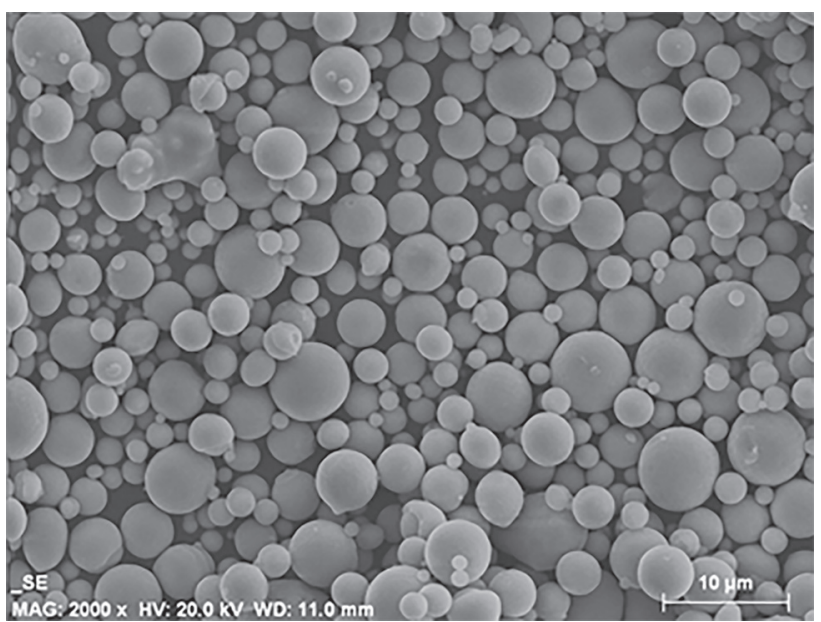

Figure 1. SEM images of the gas-atomized MnAl-C powders.

with respect to their maximum intensities, respectively. The gas-atomized MnAl-C powders are composed of $\varepsilon$ as the major phase and $\gamma_{2}$ as the minor phase, whereas the annealed powders were composed of the $\tau$-phase and a small fraction of $\gamma_{2}$-phase. The appearance of $\gamma_{2}$ was ascribed to the inhomogeneity of the MnAl-C melt and/or possibly the decomposition of $\varepsilon$-phase during the cooling process. The diffraction intensities of the $\gamma_{2}$-phase are comparable in the original and the annealed gas-atomized powders, indicating the effectiveness of the annealing process during which no additional $\gamma_{2}$-phase was formed. The structural stabilization effect of carbon in the $\tau-\mathrm{MnAl}-\mathrm{C}$ is also helpful in preventing the decomposition of the metastable $\tau$-phase at elevated temperatures.

Our previous work showed that the high purity $\varepsilon$-phase of the MnAl-C master alloys for gas-atomization can also be produced by water quenching from $1423 \mathrm{~K}$ [8]. The reflections of the water-quenched samples can be identified as pure $\varepsilon$ without any XRD-detectable $\gamma_{2}$ - or $\beta$-phases, owing to the high homogeneity of the annealed MnAl-C before quenching and high cooling rate during quenching. It is interesting that the XRD patterns of the $\varepsilon$-phase produced by gas-atomization are quite different from that produced by water quenching, as shown in figure 2 . The gas-atomized powders show strong (101) reflections, while the intensities of the other weaker reflections do not vary too much from that of the (101) reflections, indicating that the gas-atomized $\varepsilon$ is macroscopically isotropic. However, the water-quenched $\varepsilon$-phase shows much stronger (002) reflections whereas most of the other reflections are very weak, indicating that the water-quenched $\varepsilon$ is macroscopically anisotropic. It is reasonable for the tiny spherical melt droplets to form isotropic $\varepsilon$ when quenched in an isotropic manner. The mechanisms for the formation of the anisotropic water-quenched $\varepsilon$ is still unclear but may be related to the high temperature homogenization of the bulk MnAl-C before quenching, during which the grain growth 


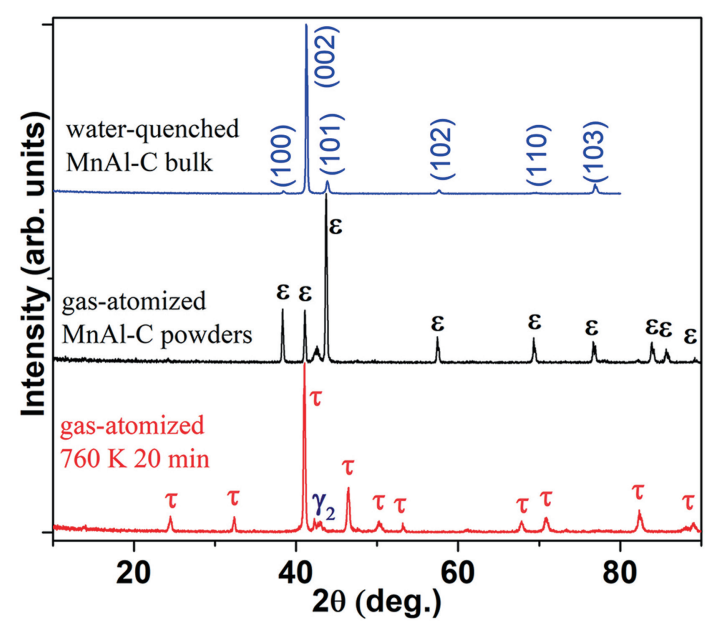

Figure 2. XRD patterns of the gas-atomized MnAl-C powders could be indexed with $\varepsilon$-phase, which transformed into $\tau$-phase when annealed at $760 \mathrm{~K}$ for $20 \mathrm{~min}$. A small fraction of $\gamma_{2}$-phase was detected in the gas-atomized powders. The mother alloys for gasatomization are $\varepsilon$-phase also after water quenching from $1423 \mathrm{~K}$.

along the $c$-axis of $\varepsilon$ is enhanced while that along the direction perpendicular to the $c$-axis is limited [8]. The diffraction peaks of the gas-atomized $\varepsilon$ were observed to shift to lower angles than those of the water-quenched $\varepsilon$, indicating larger lattice parameters of the gas-atomized $\varepsilon$-phase. The peak shift can be caused by the existence of residual strains after quenching, or the dissolution of impurity elements in the lattice. The precursors of the $\varepsilon$ obtained by water-quenching and gasatomization are bulk MnAl-C (1423 K) and MnAl-C melt $(>1500 \mathrm{~K})$, respectively. A higher temperature indicates a larger lattice parameter, which might be maintained to low temperatures by rapid quenching. Moreover, the spherical droplets were cooled down from the surface to the central part uniformly from every direction. Thus, solid surface shells would be formed prior to the solidification of central melt. The shrinkage of the central melt during solidification might generate residual strain, thereby resulting in the larger lattice parameters.

\subsection{Structural evolution detected by $M-T$ curves}

The annealing temperature is crucial for the preparation of the high purity ferromagnetic $\tau$-phase from the non-magnetic $\varepsilon$-MnAl-C. A lower temperature may result in an incomplete phase transformation via the displacive mode, while a higher temperature may result in the decomposition of the metastable $\tau$-phase [8]. We have recently developed a novel method to determine the exact phase transformation temperature by measuring the temperature dependence of magnetization of the $\varepsilon$ under an applied magnetic field [17], based on which the optimum annealing temperatures for $\varepsilon \rightarrow \tau$ transformation were selected. Figure 3 shows the temperature dependence of magnetization of the gas-atomized $\varepsilon$ powders under $2 \mathrm{~T}$ with increasing temperature from $300 \mathrm{~K}$. However, the $M$ of the $\varepsilon$-phase increases abruptly at temperatures above $720 \mathrm{~K}$, at which the massive $\varepsilon \rightarrow \tau$ phase transformation was triggered. The phase transformation mechanisms in MnAl-based magnets have been studied systematically in our previous work [8]. The massive growth of $\tau$ from $\varepsilon$ of $\mathrm{MnAl}-\mathrm{C}$ is a diffusional process involving the migration of incoherent heterophase interfaces by random attachment of atoms across the growth interface. The $M$ decreases again at $T \geq 806$ $\mathrm{K}$, owing to the accomplishment of the $\varepsilon \rightarrow \tau$ phase transformation and/or the decomposition of $\tau$-phase. It should be noted that the starting and ending temperatures of the massive phase transformation in the water-quenched $\varepsilon$ with the same composition are 766 and $838 \mathrm{~K}$, respectively [8]. It is interesting that the gas-atomized $\varepsilon$ shows lower phase transformation temperatures than that of the water-quenched $\varepsilon$. Our previous work showed that the carbon concentration in MnAl has a substantial effect on the phase transformation temperatures [17]. This work shows that the $\varepsilon$-phases with the same composition, but prepared via different routes might also exhibit different phase transformation temperatures. The difference between the XRD patterns of the $\varepsilon$ prepared by water-quenching and gas-atomization provides some evidence for the reason of the different phase transformation temperatures. As mentioned above, the larger lattice parameters of the gas-atomized $\varepsilon$ than that of the water-quenched $\varepsilon$ indicate larger micro-strains in the lattices of the gas-atomized $\varepsilon$-phase. The energy stored in the quenched lattices due to large strains might release at elevated temperatures, and thus, the temperatures for re-arrangement of the atoms during phase transformation were lowered. The activation energy for diffusional growth of $\tau$-phase in bulk water-quenched MnAl-C with the same composition had been previously estimated to be $182.5 \mathrm{~kJ} \mathrm{~mol}^{-1}$ [8]. The lower phase transformation temperature of the gas-atomized $\varepsilon$ than that of the water-quenched $\varepsilon$ indicates possible lower activation energy in the $\varepsilon$-powders. We speculate that the impurities in the powders may also be the one reason for the lower temperature/activation energy for phase transformation. Moreover, the anisotropic (or isotropic) $\varepsilon$ obtained by water-quenching (or gas-atomization) might grow into $\tau$ in an anisotropic (or isotropic) manner. The higher energy barriers for anisotropic growth of $\varepsilon \rightarrow \tau$ require a higher temperature for triggering the phase transformation. The higher growth rate for isotropic growth of $\varepsilon \rightarrow \tau$ indicates a lower accomplishing temperature for phase transformation.

Figure 3 shows that the $\mathrm{d} M / \mathrm{d} T$ in $460-720 \mathrm{~K}$ is lower than that in 300-460 K, owing to the displacive phase transformation of $\varepsilon \rightarrow \tau$ in the gas-atomized powders. The displacive phase transformation is a diffusionless process involving cooperative movements of atoms which are not hindered by large energy barriers. It is interesting that the displacive phase transformation of the gas-atomized $\varepsilon$ as shown in figure 3 is not as evident as that observed in the water-quenched $\varepsilon-\mathrm{MnAl}-\mathrm{C}$ [8]. Our previous work showed that the displacive phase transformation rate is low and the maximum size of 


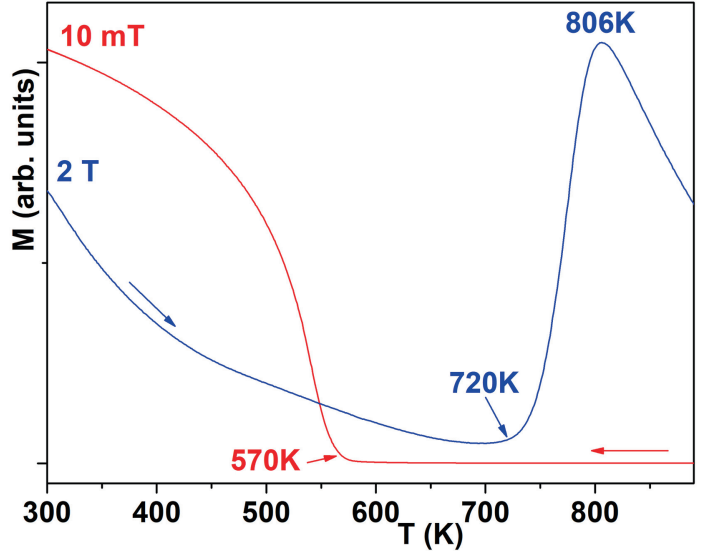

Figure 3. $M-T$ plots of the gas-atomized MnAl-C powders were recorded under an applied field of $2 \mathrm{~T}$ with increasing temperature and $10 \mathrm{mT}$ with decreasing temperature, respectively. The $\varepsilon$-phase transformed into $\tau$ phase at temperatures above $720 \mathrm{~K}$. The Curie temperature of $\tau-\mathrm{MnAl}-\mathrm{C}$ was determined to be $\sim 570 \mathrm{~K}$.

the $\tau$ formed via this mode is usually not more than $40 \mathrm{~nm}$. In comparison with the water-quenched $\varepsilon$, the gas-atomized $\varepsilon$ shows larger strains that usually result in a higher density of defects, which would probably increase the energy barriers for the diffusionless displacive phase transformation. Thus, the phase transformation rate and the grain size of $\tau$ formed via the displacive mode would be decreased, resulting in a less pronounced displacive phase transformation temperature in the gas-atomized $\varepsilon$. Figure 3 also shows that the Curie temperature of the gas-atomized $\tau-\mathrm{MnAl}-\mathrm{C}$ is in the vicinity of $570 \mathrm{~K}$.

\subsection{Microstructure of compacted MnAl-C}

Figure 4 shows the microstructure of the bulk MnAl-C prepared by high pressure compaction of the gas-atomized powders. It is interesting that the ideal spherical particles as observed in figure 1 become elongated two-dimensional plates stacking along the direction of compaction. The thickness of the plates falls in the range of $200 \mathrm{~nm}$ to typically around $1 \mu \mathrm{m}$, depending on the sizes of the original gasatomized particles. Elemental analysis shows that the black areas in figure 4a generally have a higher oxygen content, which were attributed to the surface oxidation of the particles after exposure to air. The black lines as marked by $1-5$ in figure 4 show the interfaces of large particles after cold compaction. The grain size of the particles is typically in the range of $10-100 \mathrm{~nm}$, as shown in figure $4 \mathrm{~b}$. High pressure deformation is effective in reducing the grain size and enhancing the grain boundaries of the MnAl-C bulk samples. The density of the compacted $\mathrm{MnAl}-\mathrm{C}$ is measured to be $4.91 \mathrm{~g} \mathrm{~cm}^{-3}$, which is $\sim 98.6 \%$ of the full density of the melt cast bulk alloys with the same composition.
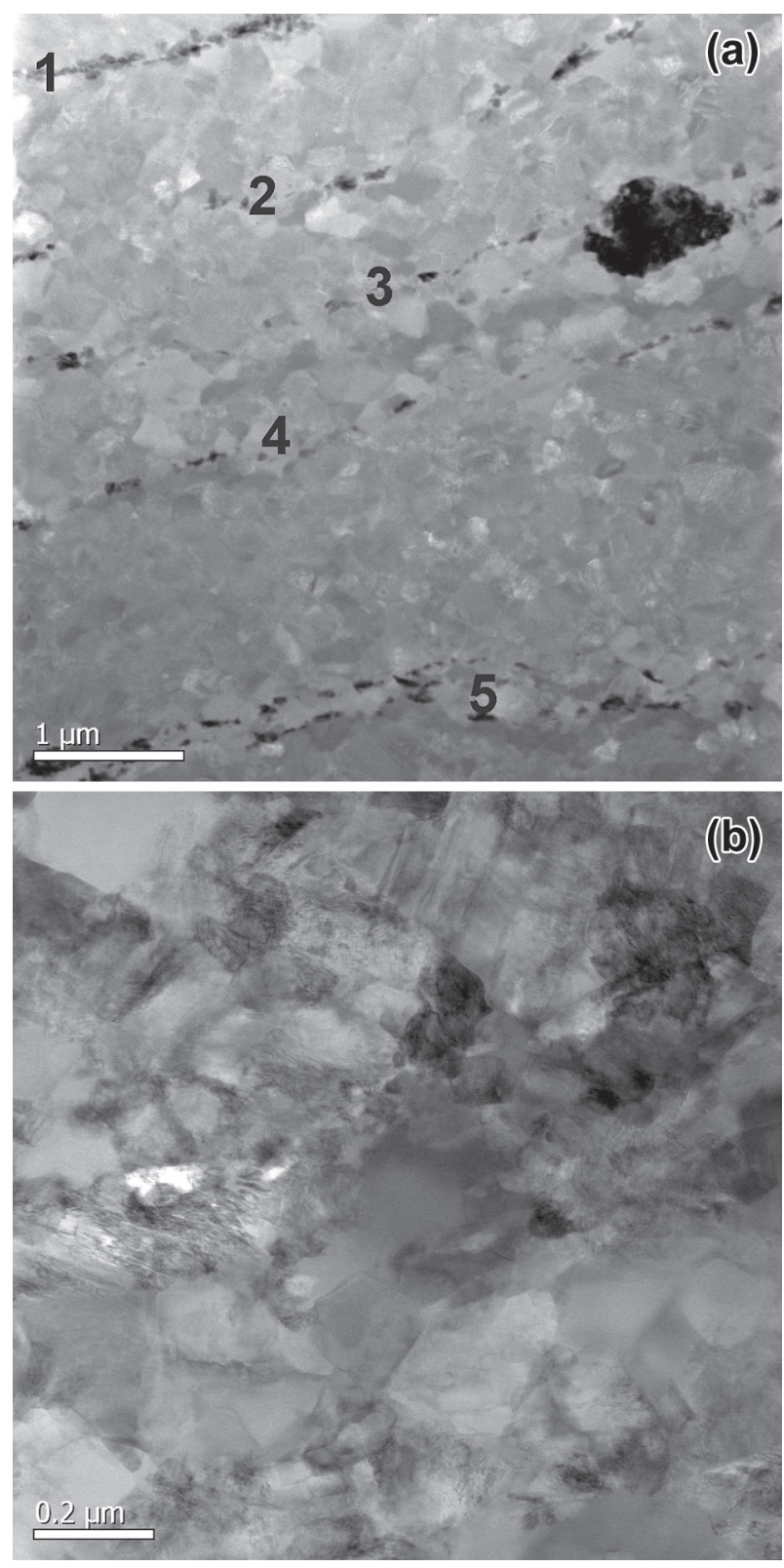

Figure 4. Typical TEM images of the bulk MnAl-C obtained by high pressure compaction of the $\tau$-phase powders. (a) The spherical particles become elongated two-dimensional plates stacking along the compaction direction. The black lines marked with $1-5$ show the interfaces of the spherical powders after compaction. (b) An enlarged image shows that the grain size in the cold deformed MnAl-C falls in the range of $10-100 \mathrm{~nm}$.

\subsection{Magnetic properties}

Figure 5 shows the magnetic hysteresis loops of the gas-atomized MnAl-C powders annealed at $760 \mathrm{~K}$ for 20 min and the bulk magnets prepared from the powders under a pressure of 1.6 and $3.1 \mathrm{GPa}$, respectively. The room temperature magnetization of the $\tau$-powders measured at $8 \mathrm{~T}$ reached up to $\sim 99 \mathrm{Am}^{2} \mathrm{~kg}^{-1}$, which is higher than those of the 


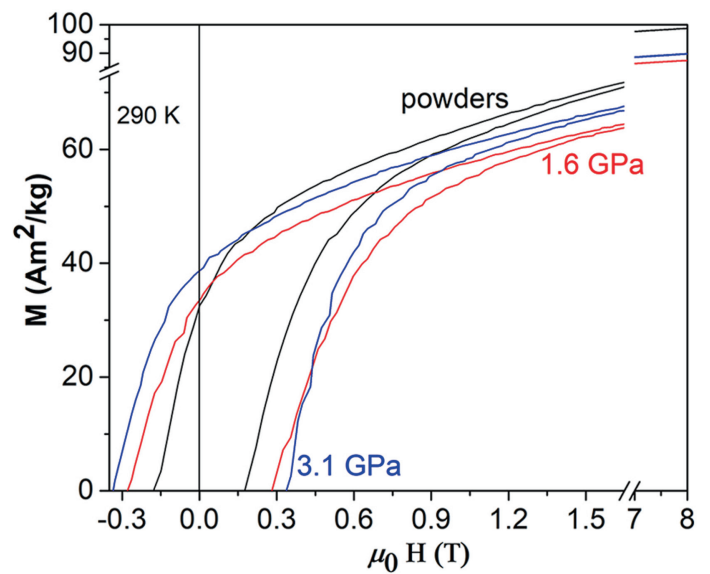

Figure 5. $M-H$ plots of the MnAl-C gas-atomized powders annealed at $760 \mathrm{~K}$ for $20 \mathrm{~min}$, the bulk magnetic materials prepared from the $\tau$-phase powders under a pressure of 1.6 and $3.1 \mathrm{GPa}$, respectively.

gas-atomized MnAl-based powders reported previously [9, $10,19]$, indicating the effectiveness of our process for producing $\tau$ from $\varepsilon$. This value is slightly lower than that of the bulk $\tau$ produced by casting and annealing methods, owing partially to the presence of a small fraction of $\gamma_{2}$ in the gasatomized powders [8]. However, this value is higher than that of most powders produced by ball-milling [9,20]. The magnetization of the high-purity bulk $\tau$-phase $\mathrm{MnAl}-\mathrm{C}$ had been reported to be $114 \mathrm{Am}^{2} \mathrm{~kg}^{-1}$ at $4 \mathrm{~T}$ [8]. In this work, the magnetization of the $\tau$-phase MnAl-C powders was measured to be $\sim 91 \mathrm{Am}^{2} \mathrm{~kg}^{-1}$ at $4 \mathrm{~T}$. Therefore, the fraction of the $\tau$ phase in our annealed powders is estimated to be $80 \mathrm{wt} . \%$, while the other non-magnetic phases accounts $20 \mathrm{wt} . \%$. Note that the surface effect in the powders may reduce the fraction of $\tau$-phase in our annealed powders. It is interesting that the magnetization of the cold-compacted magnets is in the range of $88-90 \mathrm{Am}^{2} \mathrm{~kg}^{-1}$, which is $\sim 10$ percent lower than that of the precursors. The $\sim 10 \%$ magnetization reduction in the high-pressure compaction process is much lower than that in the ball-milling process and the hot-deformation process $[21,22]$.

The coercivity of the gas-atomized powders of $\tau$-phase is $\sim 0.17 \mathrm{~T}$. The $H_{\mathrm{c}}$ of the dense magnets prepared from the $\tau$-phase powders under the pressure of $\sim 1.6$ and $\sim 3.1 \mathrm{GPa}$ was significantly enhanced and reached up to 0.28 and 0.34 $\mathrm{T}$, respectively. The enhanced coercivity in the high-pressure compacted MnAl-C was ascribed to the accumulation of dislocation and grain refinement during cold compaction. Bittner et al [16] studied the impact of dislocations on coercivity and obtained a coercivity of $0.29 \mathrm{~T}$ in the cold-worked $\mathrm{MnAl}$ and found that the defect density in the samples was significantly enhanced after cold-working. The stress fields of dislocations lead to local change of the crystallographic properties and such regions are most likely to act as pinning sites for magnetic domain walls [16]. The $M_{\mathrm{r}}$ of the gas-atomized powders of $\tau$ is $\sim 33 \mathrm{Am}^{2} \mathrm{~kg}^{-1}$, which was enhanced after high pressure compaction and reached up to $\sim 39 \mathrm{Am}^{2} \mathrm{~kg}^{-1}$ at $3.1 \mathrm{GPa}$.

\section{Conclusion}

Dense MnAl-C bulk magnetic materials were prepared by high-pressure compaction of the ferromagnetic $\tau$-phase prepared by annealing the gas-atomized powders. The resulting materials showed remarkable differences in microstructure and magnetic properties. The grain size is reduced whereas the coercivity and remanent magnetization are enhanced significantly after compaction, owing to the enlarged dislocation density. The triggering and accomplishing temperatures for the phase transformations of the $\varepsilon$ gas-atomized powders were measured, based on which an optimum annealing temperature for preparation of high-performance $\tau$-phase was selected. We found that the phase transformation temperatures of the gasatomized powders are lower than that of the water-quenched samples with the same composition.

\section{Acknowledgements}

This work was supported by the Future Materials Discovery Program through the National Research Foundation of Korea (NRF) funded by the Ministry of Science, ICT, and Future Planning (2016M3D1A1027835). P Z Si and H L Ge are grateful to the National Natural Science Foundation of China (nos. 11074227 and 51671177). China Jiliang University and Korea Institute of Materials Science have equal rights to the work.

\section{References}

[1] Hindrichs G 1908 Z. Anorg. Chem. 59414

[2] Kono H 1958 J. Phys. Soc. Jpn. 131444

[3] Koch A J, Hokkeling P, van der Steeg M G and de Vos K J 1960 J. Appl. Phys. 31 S75

[4] Zhang C, Zhang T, Wang J, Zhao S, Wu Y and Jiang C 2018 Scr. Mater. 14372

[5] Nieves P, Arapan S, Schrefl T and Cuesta-Lopez S 2017 Phys. Rev. B 96224411

[6] Thielsch J, Bittner F and Woodcock T G 2017 J. Magn. Magn. Mater. 42625

[7] Shao Z, Zhao H, Zeng J, Zhang Y, Yang W, Lai Y et al 2017 AIP Adv. 7056213

[8] Si P Z, Qian H D, Choi C J, Park J, Han S, Ge H L et al 2017 Materials 101016

[9] Lee J G, Wang X L, Zhang Z D and Choi C J 2011 Thin Solid Films 5198312

[10] Law J Y, Rial J, Villanueva M, López N, Camarero J, Marshall L G et al 2017 J. Alloys Compd. 712373

[11] Ohtani T, Kato N, Kojima S, Kojima K, Sakamoto Y, Konno I et al 1977 IEEE Trans. Magn. 131328

[12] Madugundo R, Alkan O K and Hadjipanayis G C 2016 AIP $A d v .6056009$ 
[13] Pasko A, Mazaleyrat F, Varga L K, Stamenov P $\mathrm{S}$ and Coey J M D 2014 IEEE Trans. Magn. 50 6971500

[14] Chaturvedi A, Yaqub R and Baker I 2014 Metals 420

[15] Tyrman M, Ahmim S, Pasko A, Etgens V, Mazaleyrat F, Weben S Q et al 2018 AIP Adv. 8056217

[16] Bittner F, Freudenberger J, Schultz L and Woodcock T G 2017 J. Alloys Compd. $\mathbf{7 0 4} 528$

[17] Si P Z, Qian H D, Choi C J, Park J and Ge H L 2018 J. Magn. Magn. Mater. 451540
[18] Wang X, Lee J, Lee J, Kim H, Choi C and Zhang Z 2012 Met. Mater. Int. 18711

[19] Chaturvedi A, Yaqub R and Baker I 2014 J. Phys.: Condens. Matter 26064201

[20] Singh N, Mudgil V, Anand K, Srivastava A K, Kotnala R K and Dhar A 2015 J. Alloys Compd. 633401

[21] Palanisamy D, Madras G and Chattopadhyay K 2017 J. Magn. Magn. Mater. 439181

[22] Madugundo R and Hadjipanayis G C 2016 J. Appl. Phys. 119 013904 